\title{
GENERAL DEFECT RELATIONS OF HOLOMORPHIC CURVES
}

\author{
BY \\ KIYOSHI NIINO \\ Dedicated to Professor Yûsaku Komatu on his 70th birthday
}

\begin{abstract}
Let $x$ : $\mathbf{C} \rightarrow P_{n} \mathbf{C}$ be a holomorphic curve of finite lower order $\mu$, and let $A=\{\alpha\}$ be an arbitrary finite family of holomorphic curves $\alpha: \mathbf{C} \rightarrow$ $\left(P_{n} \mathbf{C}\right)^{*}$ satisfying $T(r, \alpha)=o(T(r, x))(r \rightarrow \infty)$. Suppose $x$ is nondegenerate with respect to $A$, and $A$ is in general position. We show the following general defect relations: (1) $x$ has at most $n$ deficient curves in $A$ if $\mu=0$. (2) $\sum_{\alpha \in A} \delta(\alpha) \leq n$ if $0<\mu \leq 1 / 2$. (3) $\sum_{\alpha \in A} \delta(\alpha) \leq[2 n \mu]+1$ if $1 / 2<\mu<+\infty$.
\end{abstract}

1. Introduction. Let $f$ be a transcendental meromorphic function on the complex plane $\mathbf{C}$ and let $a_{j}(j=1,2, \ldots, q)$ be distinct meromorphic functions on C satisfying

$$
T\left(r, a_{j}\right)=o(T(r, f)), \quad r \rightarrow \infty .
$$

Then the deficiency of $a_{j}$ with respect to $f$ is defined by

$$
\delta\left(a_{j}, f\right)=1-\limsup _{r \rightarrow \infty} \frac{N\left(r, 0, f-a_{j}\right)}{T(r, f)},
$$

and $a_{j}$ is called a deficient function of $f$ when $\delta\left(a_{j}, f\right)>0$.

In 1929 Nevanlinna [10] proved that the defect relation

$$
\sum_{j=1}^{q} \delta\left(a_{j}, f\right) \leq 2
$$

is valid for $q=3$ and asked if (1.1) is true for all positive integers $q$. In 1939 Dufresnoy [4] showed that $\sum_{j=1}^{q} \delta\left(p_{j}, f\right) \leq 2+d$ for distinct polynomials $p_{j}$ of degree $\leq d$. In 1964 Chuang [3] proved $(1.1)$ when $\delta(\infty, f)=1$. Recently, Yang $[\mathbf{1 7}]$ gave the following:

THEOREM A. Let $\mu$ be the lower order of $f$.

(1) If $\mu=0, f$ has at most one deficient function (cf. Mori [8, Theorem 1]).

(2) If $\mu>0$ then

$$
\sum_{j=1}^{q} \delta\left(a_{j}, f\right) \begin{cases}\leq 1 & (0<\mu \leq 1 / 2, q=1), \\ <1-\cos \pi \mu & (0<\mu \leq 1 / 2, q \geq 2), \\ \leq 2-\sin \pi \mu & (1 / 2<\mu \leq 1), \\ \leq \min \{[2 \mu]+1,(\sqrt{2} / 2) \pi \mu\} & (\mu>1) .\end{cases}
$$

On the other hand, Mori [9] regarded $\delta\left(a_{j}, f\right)$ as a deficiency of the moving divisor and extended the above result of Nevanlinna to the case of holomorphic

Received by the editors March 5, 1984 and, in revised form, July 10, 1984.

1980 Mathematics Subject Classification. Primary 30D35.

Key words and phrases. Holomorphic curve, Nevanlinna theory, defect relation, spread relation, deficient curve. 
mappings of $\mathbf{C}^{n}$ into $P_{m} \mathbf{C}$. Further, under some conditions, Shiffman [12], Mori $[\mathbf{9}]$, and Stoll $[\mathbf{1 3}]$ obtained general defect relations for moving divisors with respect to meromorphic functions on $\mathbf{C}^{n}$, holomorphic mappings of $\mathbf{C}^{n}$ into $P_{m} \mathbf{C}$, and holomorphic mappings of $M$ into $P_{m} \mathbf{C}$, respectively, where $M$ is a parabolic complex manifold.

The purpose of this paper is to extend Theorem A to the case of holomorphic curves. We assume the reader is familiar with Nevanlinna theory of meromorphic functions and holomorphic curves (cf. $[\mathbf{1 0}, \mathbf{1 5}, \mathbf{1 6}])$.

The author is very thankful to Professors S. Mori and J. Noguchi for helpful discussions and valuable advice and is also indebted to the referee for many comments and suggestions.

2. Notations and main results. We denote complex projective $n$-space by $P_{n} \mathbf{C}$ and dual complex projective $n$-space by $\left(P_{n} \mathbf{C}\right)^{*}$. Let $x: \mathbf{C} \rightarrow P_{n} \mathbf{C}$ be a holomorphic curve and $\tilde{x}=\left(x_{0}, x_{1}, \ldots, x_{n}\right): \mathbf{C} \rightarrow \mathbf{C}^{n+1}-\{0\}$ its reduced representation. We define the characteristic (order) function $T(r, x)$ of $x$ by

$$
T(r, x)=\frac{1}{2 \pi} \int_{0}^{2 \pi} \log \left|\tilde{x}\left(r e^{i \theta}\right)\right| d \theta-\log |\tilde{x}(0)|,
$$

where $|\tilde{x}(z)|=\left(\sum_{j=0}^{n}\left|x_{j}(z)\right|^{2}\right)^{1 / 2}$.

Let $\alpha: \mathbf{C} \rightarrow\left(P_{n} \mathbf{C}\right)^{*}$ be a holomorphic curve and $\tilde{\alpha}=\left(\alpha_{0}, \alpha_{1}, \ldots, \alpha_{n}\right): \mathbf{C} \rightarrow$ $\left(\mathbf{C}^{n+1}\right)^{*}-\{0\}$ its reduced representation. We denote by $N(r, \alpha) \equiv N(r, \alpha ; x)$ the counting function of zeros of the entire function

$$
F(z) \equiv\langle\tilde{x}(z), \tilde{\alpha}(z)\rangle=\sum_{j=0}^{n} \alpha_{j}(z) x_{j}(z) \not \equiv 0,
$$

that is, $N(r, \alpha)=N(r, 0, F)$. We define the deficiency $\delta(\alpha) \equiv \delta(\alpha, x)$ of $\alpha$ with respect to $x$ by

$$
\delta(\alpha)=1-\limsup _{r \rightarrow \infty} \frac{N(r, \alpha)}{T(r, \alpha)+T(r, x)} .
$$

Since $|F(z)| \leq|\tilde{\alpha}||\tilde{x}|,(2.1)$ and Jensen's formula imply $0 \leq \delta(\alpha) \leq 1$. If $\delta(\alpha)>0$ then we say that $\alpha$ is a deficient curve. We remark that if $\alpha$ satisfies

$$
T(r, \alpha)=o(T(r, x)), \quad r \rightarrow \infty
$$

then $(2.2)$ is reduced to

$$
\delta(\alpha)=1-\limsup _{r \rightarrow \infty} \frac{T(r, \alpha)}{T(r, x)} .
$$

Now let $\alpha^{(k)}: \mathbf{C} \rightarrow\left(P_{n} \mathbf{C}\right)^{*}(k=1,2, \ldots, q ; q>n+1)$ be holomorphic curves and $\tilde{\alpha}^{(k)}=\left(\alpha_{0}^{(k)}, \alpha_{1}^{(k)}, \ldots, \alpha_{n}^{(k)}\right): \mathbf{C} \rightarrow\left(\mathbf{C}^{n+1}\right)^{*}-\{0\}$ their reduced representation. If $\operatorname{det}\left\{\left(\alpha^{\left(k_{i}\right)}(z)\right)_{1 \leq i \leq n+1,0 \leq j \leq n}\right\} \not \equiv 0$ for any $\left\{k_{1}, k_{2}, \ldots, k_{n+1}\right\} \subset\{1,2, \ldots, q\}$, then we say that $\left\{\alpha^{(k)}\right\}_{k=1}^{q}$ is in general position. A curve $x: \mathbf{C} \rightarrow P_{n} \mathbf{C}$ is called nondegenerate with respect to $\left\{\alpha^{(k)}\right\}$ if

$$
F_{k}(z)=\sum_{j=0}^{n} \alpha_{j}^{(k)}(z) x_{j}(z) \not \equiv 0 \quad \text { for all } k .
$$

Using Yang's idea [17], we prove the following defect relations: 
THEOREM 1. Let $x: \mathbf{C} \rightarrow P_{n} \mathbf{C}$ be a holomorphic curve of lower order $\mu$ and $A=\{\alpha\}$ an arbitrary finite family of holomorphic curves $\alpha: \mathbf{C} \rightarrow\left(P_{n} \mathbf{C}\right)^{*}$ satisfying

$$
T(r, \alpha)=o(T(r, x)), \quad r \rightarrow \infty .
$$

Suppose $x$ is nondegenerate with respect to $A$, and $A$ is in general position. Then:

(I) If $\mu=0$ there are at most $n$ deficient curves in $A$.

(II) Assume $0<\mu \leq 1 / 2$ and put $B=\{\alpha \in A ; \delta(\alpha)>1-\cos \pi \mu\}$ and $C=$ $\{\alpha \in A ; \delta(\alpha)=1-\cos \pi \mu\}$. If there are $n$ curves belonging to $B \cup C$, then all the remaining deficiencies are equal to zero. If $\# B=p<n$, then

$$
\sum_{\alpha \in A-B} \delta(\alpha) \leq(n-p)(1-\cos \pi \mu),
$$

where equality holds if and only if $\# C=n-p$ and $\delta(\alpha)=0$ for all $\alpha \in A-(B \cup C)$.

(III) If $1 / 2<\mu<+\infty$ we have

$$
\sum_{\alpha \in A} \delta(\alpha) \leq[2 n \mu]+1-\cos (2 n \mu-[2 n \mu])(\pi / 2)
$$

As an immediate consequence of (I) and (II) we have

COROLlaRY. If $0 \leq \mu \leq 1 / 2$, then $\sum_{\alpha \in A} \delta(\alpha) \leq n$.

3. Spread relation. Let $x$ : $\mathbf{C} \rightarrow P_{n} \mathbf{C}$ be a holomorphic curve of lower order $\mu(0<\mu<+\infty)$ and $\tilde{x}=\left(x_{0}, x_{1}, \ldots, x_{n}\right): \mathbf{C} \rightarrow \mathbf{C}^{n+1}-\{0\}$ its reduced representation. Suppose that a holomorphic curve $\alpha: \mathbf{C} \rightarrow\left(P_{n} \mathbf{C}\right)^{*}$ and its reduced representation $\tilde{\alpha}: \mathbf{C} \rightarrow\left(\mathbf{C}^{n+1}\right)^{*}-\{0\}$ satisfy

$$
T(r, \alpha)=o(T(r, x)), \quad r \rightarrow \infty,
$$

and $\langle\tilde{x}(z), \tilde{\alpha}(z)\rangle \not \equiv 0$. We put

$$
\|x(z), \alpha(z)\|=|\langle\tilde{x}(z), \tilde{\alpha}(z)\rangle| /(|\tilde{x}(z)||\tilde{\alpha}(z)|) .
$$

A positive, increasing, unbounded sequence $\left\{r_{m}\right\}$ is called a sequence of Pólya peaks of order $\mu$ of $T(r, x)$ if it is possible to find positive sequences $\left\{r_{m}^{\prime}\right\},\left\{r_{m}^{\prime \prime}\right\}$, and $\left\{\varepsilon_{m}\right\}$ such that, as $m \rightarrow \infty$, then $r_{m}^{\prime} \rightarrow \infty, r_{m} / r_{m}^{\prime} \rightarrow \infty, r_{m}^{\prime \prime} / r_{m} \rightarrow \infty, \varepsilon_{m} \rightarrow 0$, and

$$
T(t, x) / T\left(r_{m}, x\right) \leq\left(t / r_{m}\right)^{\mu}\left(1+\varepsilon_{m}\right) \quad\left(r_{m}^{\prime}<t<r_{m}^{\prime \prime}\right) .
$$

We now define the spread $\sigma(\alpha) \equiv \sigma(\alpha, x)$ of $\alpha$ with respect to $x$. Let $\left\{r_{m}\right\}$ be a sequence of Pólya peaks of order $\mu$ of $T(r, x)$ and $\Lambda(r)$ a positive function satisfying

$$
\Lambda(r)=o(T(r, x)), \quad r \rightarrow \infty .
$$

Put

$$
E_{\Lambda}(r, \alpha) \equiv E_{\Lambda}(r, \alpha ; x)=\left\{\theta ; \log \left\|x\left(r e^{i \theta}\right), \alpha\left(r e^{i \theta}\right)\right\|<-\Lambda(r)\right\} \subset(-\pi, \pi]
$$

and let

Then we define

$$
\sigma_{\Lambda}(\alpha)=\liminf _{m \rightarrow \infty} \operatorname{meas} E_{\Lambda}\left(r_{m}, \alpha\right)
$$

$$
\sigma(\alpha)=\inf _{\Lambda} \sigma_{\Lambda}(\alpha)
$$

where the infimum is taken over all functions $\Lambda(r)$ satisfying (3.3).

We obtain the following spread relation, which is a generalization of Baernstein $[\mathbf{1}]$-Yang $[\mathbf{1 7}]$ and the author $[\mathbf{1 1}]$-Krytov [7]. 
THEOREM 2 (SPREAD RELATION). Let $x: \mathbf{C} \rightarrow P_{n} \mathbf{C}$ be a holomorphic curve of lower order $\mu(0<\mu<+\infty)$. Then

$$
\sigma(\alpha) \geq \min \left\{2 \pi,(4 / \mu) \sin ^{-1}(\delta(\alpha) / 2)^{1 / 2}\right\}
$$

for every holomorphic curve $\alpha: \mathbf{C} \rightarrow\left(P_{n} \mathbf{C}\right)^{*}$ satisfying (3.1) and $\|x(z), \alpha(z)\| \not \equiv 0$.

PROOF. We may assume that the reduced representations $\tilde{x}$ and $\tilde{\alpha}$ of $x$ and $\alpha$, respectively, satisfy

$$
|\tilde{x}(0)|=1 \text { and }|\tilde{\alpha}(0)|=1 \text {. }
$$

We have

$$
E_{\Lambda}(r, \alpha)=\left\{\theta ; \log \left|\tilde{x}\left(r e^{i \theta}\right)\right|+\log \left|\tilde{\alpha}\left(r e^{i \theta}\right)\right|-\log \left|F\left(r e^{i \theta}\right)\right|>\Lambda(r)\right\},
$$

where

$$
F(z)=\langle\tilde{x}(z), \tilde{\alpha}(z)\rangle=\sum_{j=0}^{n} \alpha_{j}(z) x_{j}(z) .
$$

Define the entire function $h(z)$ by

$$
h(z)=c z^{-k} F(z),
$$

where $k$ is a nonnegative integer, $c$ is a nonzero constant, and

$$
h(0)=1 \text {. }
$$

Then

$$
N(r, \alpha)=N(r, 0, F)=N(r, 0, h)+k \log r .
$$

We put

$$
\begin{gathered}
G(z)=\log |\tilde{x}(z)|+\log |\tilde{\alpha}(z)|-\log |h(z)|, \\
\Lambda_{1}(r)=\Lambda(r)+k \log r-\log |c|
\end{gathered}
$$

and

$$
E_{\Lambda_{1}}(r, G)=\left\{\theta ; G\left(r e^{i \theta}\right)>\Lambda_{1}(r)\right\}
$$

Then we deduce that

$$
E_{\Lambda_{1}}(r, G)=E_{\Lambda}(r, \alpha)
$$

and

$$
\Lambda_{1}(r)=o(T(r, x)) \quad(r \rightarrow \infty) .
$$

We next write $T(r)=T(r, x)+T(r, \alpha)$. Then it follows from (3.1) that

$$
T(r) \sim T(r, x), \quad r \rightarrow \infty,
$$

that is, $T(r) / T(r, x) \rightarrow 1$ as $r \rightarrow 1$. Hence, $T(r)$ and $T(r, x)$ have the same sequence of Pólya peaks, and, combining (3.6) and (3.10) with the definition of deficiency $\delta(\alpha)$, we have

$$
\delta(\alpha)=1-\limsup _{r \rightarrow \infty} \frac{N(r, 0, h)}{T(r)}
$$


Therefore, in order to prove Theorem 2 , from (3.8)-(3.11) it is sufficient to prove that

$$
\liminf _{r \rightarrow \infty} \operatorname{meas} E_{\Lambda_{1}}\left(r_{m}, G\right) \geq \min \left\{2 \pi, \frac{4}{\mu} \sin ^{-1}\left(\frac{\delta(\alpha)}{2}\right)^{1 / 2}\right\} .
$$

Following Baernstein [1] we now define

$$
T^{*}(z)=\sup _{E} \frac{1}{2 \pi} \int_{E} G\left(r e^{i \omega}\right) d \omega+N(r, 0, h) \quad\left(z=r e^{i \theta}, 0<r<+\infty, 0 \leq \theta \leq \pi\right)
$$

where the supremum is taken over all measurable sets $E \subset(-\pi, \pi]$ whose measure equals $2 \theta$. Further, we define

$$
T^{*}(0)=0 \text {. }
$$

Then since $\log |\tilde{x}(z)|+\log |\tilde{\alpha}(z)|$ and $\log |h(z)|$ are subharmonic, from (3.4), (3.5), (3.7), (3.13), and Theorem $\mathrm{A}^{\prime}$ in Baernstein [2], it follows that $T^{*}(z)$ is subharmonic in $\{z ; \operatorname{Im} z>0\}$ and continuous on $\{z ; \operatorname{Im} z \geq 0\}$. By definition of $T^{*}(z)$,

$$
T^{*}\left(r e^{i \pi}\right)=T^{*}(-r)=T(r), \quad T^{*}(r)=N(r, 0, h) \equiv N(r) .
$$

Since $|h(z)| \leq|c||z|^{-k}|\tilde{\alpha}(z)||\tilde{x}(z)|$, then $G(z)+\log |c|-k \log |z| \geq 0$ for all $z$. Hence, we deduce that

$$
\begin{aligned}
T^{*}\left(r e^{i \theta}\right) & \leq T^{*}\left(r e^{i \pi}\right)+((\pi-\theta) / \pi)\{\log |c|-k \log r\} \\
& <T(r)+K \quad(r>1),
\end{aligned}
$$

where $K$ is a positive constant such that $\log ^{+}|c|<K$.

Now we can apply the arguments of Baernstein $[1, \mathrm{pp} .430-433]$ and the author $[11, \mathrm{pp} .364-365]$. For the sake of clarification we sketch them here.

If $\delta(\alpha)=0$ there is nothing to prove, so we may assume $\delta(\alpha)>0$. Put

$$
\gamma=(1 / 2 \pi) \min \left\{2 \pi,(4 / \mu) \sin ^{-1}(\delta(\alpha) / 2)^{1 / 2}\right\} \text {. }
$$

Then

$$
0<\gamma \leq 1, \quad 0<\gamma \mu \leq 1 / 2 \text { and } \quad 1-\delta(\alpha) \leq \cos \pi \gamma \mu
$$

Define

$$
v(z)=T^{*}\left(z^{\gamma}\right) \quad\left(z=r e^{i \theta}, 0<r<\infty, 0 \leq \theta \leq \pi\right) .
$$

Then $v(z)$ is subharmonic in $\{z ; \operatorname{Im} z>0\}$ and continuous in $\{z ; \operatorname{Im} z \geq 0\}$. Hence, when $r e^{i \theta} \in D_{R}=\left\{z=r e^{i \theta} ; 0<r<R, 0<\theta<\pi\right\}$,

$$
v\left(r e^{i \theta}\right) \leq \int_{-R}^{R} v(t) A(t, r, \theta, R) d t+\int_{0}^{\pi} v\left(R e^{i \psi}\right) B(\psi, r, \theta, R) d \psi,
$$

where

$$
\begin{aligned}
A(t, r, \theta, R) & =\frac{1}{\pi} \frac{r \sin \theta}{t^{2}-2 t r \cos \theta+r^{2}}-\frac{1}{\pi} \frac{R^{2} r \sin \theta}{R^{4}-2 r t R^{2} \cos \theta+r^{2} t^{2}}, \\
B(\psi, r, \theta, R) & =\frac{2 R r \sin \theta}{\pi} \frac{\left(R^{2}-r^{2}\right) \sin \psi}{\left|R^{2} e^{2 i \psi}-2 r R e^{i \psi} \cos \theta+r^{2}\right|^{2}} .
\end{aligned}
$$

It follows from (3.14) and (3.15) that

$$
v(t)=T^{*}\left(t^{\gamma}\right)=N\left(t^{\gamma}\right), \quad v(-t) \leq T(r)+K, \quad v\left(R e^{i \psi}\right) \leq T(r)+K .
$$


We deduce that

$$
\begin{aligned}
& 0<A(t, r, \theta, R) \leq \frac{1}{\pi} \frac{r \sin \theta}{t^{2}-2 t r \cos \theta+r^{2}} \equiv P(t, r, \pi-\theta) \\
& (0<r<R, 0<\theta<\pi), \\
& 0<B(\psi, r, \theta, R) \leq 32 r / \pi R \quad(0<\theta<\pi, 0<\psi<\pi, 0<r<R / 2),
\end{aligned}
$$

and

$$
\int_{0}^{\infty} P(t, r, \theta) d \theta=\frac{\theta}{\pi}<1 \quad(0<\theta<\pi, r>0) .
$$

Using (3.18)-(3.21) in (3.17) we obtain

$$
\begin{aligned}
v\left(r e^{i \theta}\right) \leq & \int_{0}^{R} N\left(t^{\gamma}\right) P(t, r, \pi-\theta) d t+\int_{0}^{R} T\left(t^{\gamma}\right) P(t, r, \theta) d t \\
& +32(r / R)\left(T\left(R^{\gamma}\right)+K\right)+K \quad(0<\theta<\pi, 0<r<R / 2) .
\end{aligned}
$$

Let $\left\{r_{m}\right\}$ be any sequence of Pólya peaks of order $\mu$ of $T(r, x)$ and put $s_{m}=r_{m}^{1 / \gamma}$. $\left\{r_{m}\right\}$ is also a sequence of Pólya peaks of order $\mu$ of $T(r)$. Hence, it follows from the discussion of Baernstein [1, pp. 332-333] that

$$
v\left(s_{m} e^{i \theta}\right) \leq T\left(r_{m}\right)\left\{\cos (\pi-\theta) \gamma \mu+\alpha_{m}\right\} \quad(m=1,2, \ldots ; 0<\theta<\pi),
$$

where $\left\{\alpha_{m}\right\}$ is a sequence tending to zero. Let

$$
\sigma_{m}=\text { meas } E_{\Lambda_{1}}\left(r_{m}, G\right) \equiv \text { meas } E\left(r_{m}\right) .
$$

Then (3.12) is equivalent to the inequality

$$
\liminf _{m \rightarrow \infty} \sigma_{m} \geq 2 \pi \gamma
$$

It follows from (3.4) and (3.5) that

$$
\begin{aligned}
T\left(r_{m}\right) & =\frac{1}{2 \pi} \int_{-\pi}^{\pi}\left(\log \left|\tilde{x}\left(r_{m} e^{i \omega}\right)\right|+\log \left|\tilde{\alpha}\left(r_{m} e^{i \omega}\right)\right|\right) d \omega \\
& =\frac{1}{2 \pi} \int_{-\pi}^{\pi} G\left(r_{m} e^{i \omega}\right) d \omega+N\left(r_{m}\right) \\
& \leq \frac{1}{2 \pi} \int_{E\left(r_{m}\right)} G\left(r_{m} e^{i \omega}\right) d \omega+N\left(r_{m}\right)+\Lambda_{1}\left(r_{m}\right) \\
& \leq T^{*}\left(r_{m} e^{i \sigma_{m} / 2}\right)+\Lambda_{1}\left(r_{m}\right) .
\end{aligned}
$$

Dividing by $T\left(r_{m}\right)$ and remembering (3.15), we find that

$$
\lim _{m \rightarrow \infty} \frac{T^{*}\left(r_{m} e^{i \sigma_{m} / 2}\right)}{T\left(r_{m}\right)}=1 .
$$

Let $M=\left\{m ; \sigma_{m}<2 \pi \gamma\right\}$. If $M$ is a finite set, then (3.24) holds and we have finished, so we assume that $M$ is infinite. The point $\left(r_{m} e^{i \sigma_{m} / 2}\right)^{1 / \gamma}=s_{m} e^{i \sigma_{m} / 2 \gamma}$ belongs to the domain of $v(z)$, i.e. the upper half-plane, if and only if $m \in M$, in which case we have

$$
T^{*}\left(r_{m} e^{i \sigma_{m} / 2}\right)=v\left(s_{m} e^{i \sigma_{m} / 2 \gamma}\right) \quad(m \in M) .
$$

Using this in (3.25), comparing with (3.23), and remembering (3.16), we deduce that $\lim _{m \rightarrow \infty}, m \in M \sigma_{m} / 2 \gamma=\pi$, which shows that (3.24) holds in this case also.

Thus the proof of Theorem 2 is complete. 
4. Lemmas. In order to prove Theorem 1 we need several lemmas.

LEMMA 1. Let $x: \mathbf{C} \rightarrow P_{n} \mathbf{C}$ be a holomorphic curve, $\alpha^{(k)}: \mathbf{C} \rightarrow\left(P_{n} \mathbf{C}\right)^{*}(k=$ $1,2, \ldots, n+1)$ holomorphic curves in general position, and $\Lambda(r)$ a positive function satisfying (3.2). Suppose $x$ is nondegenerate with respect to $\left\{\alpha^{(k)}\right\}_{k=1}^{n+1}$. Then

$$
\operatorname{meas}\left\{\bigcap_{k=1}^{n+1} E_{\Lambda}\left(r, \alpha^{(k)}, x\right)\right\} \leq \frac{2 \pi}{\Lambda(r)}\left\{\sum_{k=1}^{n+1} T\left(r, \alpha^{(k)}\right)+K\right\},
$$

where $K$ is a constant independent of $\Lambda$.

Proof. Let $\tilde{x}=\left(x_{0}, x_{1}, \ldots, x_{n}\right): \mathbf{C} \rightarrow \mathbf{C}^{n+1}-\{0\}$ and

$$
\tilde{\alpha}^{(k)}=\left(\alpha_{0}^{(k)}, \alpha_{1}^{(k)}, \ldots, \alpha_{n}^{(k)}\right): \mathbf{C} \rightarrow\left(\mathbf{C}^{n+1}\right)^{*}-\{0\}
$$

be reduced representations of $x$ and $\alpha^{(k)}$, respectively. By the linear equations

$$
\sum_{j=0}^{n} \alpha_{j}^{(k)}(z) x_{j}(z)=F_{k}(z), \quad k=1,2, \ldots, n+1,
$$

we have $x_{j}=D_{j} / D$, where $D$ is the coefficient determinant of (4.1) and

$$
D_{j}=\sum_{k=1}^{n+1}(-1)^{j+k+1} D_{k j} F_{k},
$$

where $D_{k j}$ is the minor of $D$ obtained by omitting the $k$ th row and $(j+1)$ th column from $D$. The Hadamard inequality yields

$$
\left|D_{k j}\right| \leq \prod_{l=1, \neq k}^{n+1}\left|\tilde{\alpha}^{(l)}\right|
$$

so

$$
\left|x_{j}\right| \leq \frac{1}{|D|} \sum_{k=1}^{n+1}\left(\prod_{l=1, \neq k}^{n+1}\left|\tilde{\alpha}^{(l)}\right|\right)\left|F_{k}\right|
$$

Hence,

$$
\log |\tilde{x}| \leq \log \left\{\sum_{k=1}^{n+1}\left(\prod_{l=1, \neq k}^{n+1}\left|\tilde{\alpha}^{(l)}\right|\right)\left|F_{k}\right|\right\}-\log |D|+\frac{1}{2} \log (n+1) .
$$

For $z=r e^{i \theta}$, with $\theta \in E(r) \equiv \bigcap_{k=1}^{n+1} E_{\Lambda}\left(r, \alpha^{(k)}\right)$, we have

$$
\left|F_{k}(z)\right| /\left(|\tilde{x}(z)|\left|\tilde{\alpha}^{(k)}(z)\right|\right)<\exp (-\Lambda(r)), \quad k=1,2, \ldots, n+1,
$$

so

$$
\left\{\sum_{k=1}^{n+1}\left(\prod_{l=1, \neq k}^{n+1}\left|\tilde{\alpha}^{(l)}\right|\right)\left|F_{k}\right|\right\} /\left(|\tilde{x}| \prod_{k=1}^{n+1}\left|\alpha^{(k)}\right|\right)<(n+1) \exp (-\Lambda(r))
$$

Hence, combining this with (4.2) we obtain

$$
\sum_{k=1}^{n+1} \log \left|\tilde{\alpha}^{(k)}(z)\right|-\log |D(z)|>\Lambda(r)-\frac{3}{2} \log (n+1) \text { for } z=r e^{i \theta}, \theta \in E(r)
$$


The Hadamard inequality implies $|D(z)| \leq \prod_{k=1}^{n+1}\left|\tilde{\alpha}^{(k)}(z)\right|$, so

$$
\sum_{k=1}^{n+1} \log \left|\tilde{\alpha}^{(k)}(z)\right|-\log |D(z)| \geq 0
$$

for all $z$. Hence, it follows from (4.3) and (4.4) that

$$
\begin{aligned}
\frac{1}{2 \pi}( & \left.\Lambda(r)-\frac{3}{2} \log (n+1)\right) \text { meas } E(r) \\
& \leq \frac{1}{2 \pi} \int_{E(r)}\left(\sum_{k=1}^{n+1} \log \left|\tilde{\alpha}^{(k)}\left(r e^{i \theta}\right)\right|-\log \left|D\left(r e^{i \theta}\right)\right|\right) d \theta \\
& \leq \frac{1}{2 \pi} \int_{-\pi}^{\pi}\left(\sum_{k=1}^{n+1} \log \left|\tilde{\alpha}^{(k)}\left(r e^{i \theta}\right)\right|-\log \left|D\left(r e^{i \theta}\right)\right|\right) d \theta \\
& =\sum_{k=1}^{n+1}\left(T\left(r, \alpha^{(k)}\right)+\log \left|\tilde{\alpha}^{(k)}(0)\right|\right)-\left(N(r, 0, D)+\log \left|c_{\lambda}\right|\right)
\end{aligned}
$$

where

$$
D(z)=c_{\lambda} z^{\lambda}+c_{\lambda+1} z^{\lambda+1}+\cdots \quad\left(c_{\lambda} \neq 0\right) .
$$

Hence, choosing a constant $K$ such that

$$
\sum_{k=1}^{n+1} \log \left|\tilde{\alpha}^{(k)}(0)\right|-\log \left|c_{\lambda}\right|+\frac{3}{2} \log (n+1)<K,
$$

we obtain

$$
\text { meas } E(r) \leq \frac{2 \pi}{\Lambda(r)}\left\{\sum_{k=1}^{n+1} T\left(r, \alpha^{(k)}\right)+K\right\},
$$

which proves Lemma 1.

Now let $A=\left\{\alpha^{(k)}\right\}_{k=1}^{q}(q \geq n+1)$ be a finite family of holomorphic curves in general position which satisfy

$$
T\left(r, \alpha^{(k)}\right)=o(T(r, x)), \quad r \rightarrow \infty
$$

Further suppose that $x$ is nondegenerate with respect to $A$. We define $I_{\Lambda}\left(r, \alpha^{(k)}\right) \equiv$ $I_{\Lambda}\left(r, \alpha^{(k)}, A\right)$ by

$$
\begin{aligned}
I_{\Lambda}\left(r, \alpha^{(k)}\right)= & E_{\Lambda}\left(r, \alpha^{(k)}\right) \\
& -\bigcup_{\left(l_{1}, \ldots, l_{n}\right)}\left\{E_{\Lambda}\left(r, \alpha^{(k)}\right) \cap\left(\bigcap_{j=1}^{n} E_{\Lambda}\left(r, \alpha^{\left(l_{j}\right)}\right)\right)\right\},
\end{aligned}
$$

where the union is taken over all possible $n$-tuples $\left(l_{1}, \ldots, l_{n}\right) \subset(1, \ldots, k-1, k+$ $1, \ldots, q)$, and

$$
\sigma_{\Lambda}^{\prime}\left(\alpha^{(k)}\right)=\liminf _{m \rightarrow \infty} \operatorname{meas} I_{\Lambda}\left(r_{m}, \alpha^{(k)}\right)
$$


Then we can deduce

LEMMA 2. For any $\left(k_{1}, \ldots, k_{n+1}\right) \subset(1,2, \ldots, q)$ we have

$$
\bigcap_{j=1}^{n+1} I_{\Lambda}\left(r, \alpha^{\left(k_{j}\right)}\right)=\emptyset
$$

We now prove

LEMMA 3. There is a sequence of positive functions $\Lambda_{\nu}(r)$ satisfying (3.3) such that

$$
\liminf _{\nu \rightarrow \infty} \sigma_{\Lambda_{\nu}}^{\prime}\left(\alpha^{(k)}\right) \geq \min \left\{2 \pi, \frac{4}{\mu} \sin ^{-1}\left(\frac{\delta\left(\alpha^{(k)}\right)}{2}\right)^{1 / 2}\right\} .
$$

Proof. We can choose a constant $K$ such that Lemma 1 is valid for all $(n+1)$ tuples $\left(k_{1}, \ldots, k_{n+1}\right) \subset(1, \ldots, q)$. Hence, it follows that

$$
\begin{aligned}
& \sum_{\left(l_{1}, \ldots, l_{n}\right)} \operatorname{meas}\left\{E_{\Lambda}\left(r, \alpha^{(k)}\right) \cap\left(\bigcap_{j=1}^{n} E_{\Lambda}\left(r, \alpha^{\left(l_{j}\right)}\right)\right)\right\} \\
& \leq \sum_{\left(l_{1}, \ldots, l_{n}\right)} \frac{2 \pi}{\Lambda(r)}\left\{T\left(r, \alpha^{(k)}\right)+\sum_{j=1}^{n} T\left(r, \alpha^{\left(l_{j}\right)}\right)+K\right\} \\
& \leq \frac{2 \pi}{\Lambda(r)}\left(\begin{array}{c}
q-1 \\
n
\end{array}\right)\left(\sum_{l=1}^{q} T\left(r, \alpha^{(l)}\right)+K\right) .
\end{aligned}
$$

Choose a sequence of positive numbers $\varepsilon_{\nu}$ such that $\lim _{\nu \rightarrow \infty} \varepsilon_{\nu}=0$. Put

$$
\Lambda_{\nu}(r)=\frac{2 \pi}{\varepsilon_{\nu}}\left(\begin{array}{c}
q-1 \\
n
\end{array}\right)\left(\sum_{l=1}^{q} T\left(r, \alpha^{(l)}\right)+K\right) .
$$

Then it follows from (4.5) that for every fixed $\nu, \Lambda_{\nu}(r)$ satisfies (3.3). From the definition of $\sigma_{\Lambda}(\alpha)$ and (4.6)-(4.9) we deduce that, for every fixed $\nu$,

$$
\begin{aligned}
\operatorname{meas} I_{\Lambda_{\nu}}\left(r_{m}, \alpha^{(k)}\right) \geq & \operatorname{meas} E_{\Lambda_{\nu}}\left(r_{m}, \alpha^{(k)}\right) \\
& -\sum_{\left(l_{1}, \ldots, l_{n}\right)} \operatorname{meas}\left\{E_{\Lambda_{\nu}}\left(r_{m}, \alpha^{(k)}\right) \cap\left(\bigcap_{j=1}^{n} E_{\Lambda_{\nu}}\left(r_{m}, \alpha^{\left(l_{j}\right)}\right)\right)\right\} \\
\geq & \operatorname{meas} E_{\Lambda_{\nu}}\left(r_{m}, \alpha^{(k)}\right)-\varepsilon_{\nu},
\end{aligned}
$$

so

$$
\sigma_{\Lambda_{\nu}}^{\prime}\left(\alpha^{(k)}\right) \geq \sigma_{\Lambda_{\nu}}\left(\alpha^{(k)}\right)-\varepsilon_{\nu}
$$

Therefore, our spread relation implies

$$
\sigma_{\Lambda_{\nu}}^{\prime}\left(\alpha^{(k)}\right) \geq \min \left\{2 \pi, \frac{4}{\mu} \sin ^{-1}\left(\frac{\delta\left(\alpha^{(k)}\right)}{2}\right)^{1 / 2}\right\}-\varepsilon_{\nu}
$$

for every fixed $\nu$. Here let $\nu \rightarrow \infty$. Then we obtain Lemma 3. 
We deduce the following obvious

LEMMA 4. Let $E_{1}, \ldots, E_{q}$ be measurable subsets of $\{|z|=1\}$. Define $E=$ $E_{1} \cup \cdots \cup E_{q}$. Take an integer $p$ with $1 \leq p \leq q$. Assume that for any selection of integers $1 \leq i_{1}<i_{2}<\cdots<i_{p} \leq q$, we have $E_{i_{1}} \cap E_{i_{2}} \cap \cdots \cap E_{i_{p}}=\emptyset$. Then

$$
\sum_{j=1}^{q} \operatorname{meas} E_{j} \leq(p-1) \text { meas } E \text {. }
$$

We finally prove

LEMMA 5. Let $x: \mathbf{C} \rightarrow P_{n} \mathbf{C}$ be a holomorphic curve of lower order $\mu(0<$ $\mu<+\infty)$ and $\alpha^{(k)}: \mathbf{C} \rightarrow\left(P_{n} \mathbf{C}\right)^{*}, k=1, \ldots, q(q \geq n+1)$ holomorphic curves satisfying (4.5). Suppose that $x$ is nondegenerate with respect to $\left\{\alpha^{(k)}\right\}$, and $\left\{\alpha^{(k)}\right\}$ is in general position. Then

$$
\sum_{k=1}^{q} \min \left\{2 \pi, \frac{4}{\mu} \sin ^{-1}\left(\frac{\delta\left(\alpha^{(k)}\right)}{2}\right)^{1 / 2}\right\} \leq 2 n \pi .
$$

ProOF. Lemmas 2 and 4 imply

$$
\sum_{k=1}^{q} \operatorname{meas} I_{\Lambda}\left(r, \alpha^{(k)}\right) \leq 2 n \pi
$$

and, consequently,

$$
\sum_{k=1}^{q} \sigma_{\Lambda}^{\prime}\left(\alpha^{(k)}\right) \leq 2 n \pi
$$

Hence, we deduce from Lemma 3 that

$$
\sum_{k=1}^{q} \min \left\{2 \pi, \frac{4}{\mu} \sin ^{-1}\left(\frac{\delta\left(\alpha^{(k)}\right)}{2}\right)^{1 / 2}\right\} \leq 2 n \pi,
$$

which proves Lemma 5 .

5. Proof of Theorem $\mathbf{1}(\mathrm{I})$. Theorem $1(\mathrm{I})$ is an immediate consequence of the Corollary to Theorem 3, which is a generalization of results of Edrei-Fuchs [6], Toda [14], and Mori $[\mathbf{8}]$ (cf. Yang [17]).

THEOREM 3. Let $x: \mathbf{C} \rightarrow P_{n} \mathbf{C}$ be a holomorphic curve of lower order $\mu$ and $\alpha^{(k)}: \mathbf{C} \rightarrow\left(P_{n} \mathbf{C}\right)^{*}(k=1, \ldots, n+1)$ holomorphic curves satisfying $T\left(r, \alpha^{(k)}\right)=$ $o(T(r, x))(r \rightarrow \infty)$. Assume that $x$ is nondegenerate with respect to $\left\{\alpha^{(k)}\right\}_{k=1}^{n+1}$, $\left\{\alpha^{(k)}\right\}_{k=1}^{n+1}$ is in general position, and $\alpha^{(k)}(k=1, \ldots, n+1)$ are deficient curves with respect to $x$. If $\gamma=\max _{1 \leq k \leq n+1}\left\{1-\delta\left(\alpha^{(k)}\right)\right\}$, then

$$
\mu \geq \begin{cases}\frac{\log (1 / \gamma(2-\gamma))}{\log (1+4 / \gamma(1-\gamma))} & (\gamma \neq 0) \\ 1 & (\gamma=0) .\end{cases}
$$

COROLLARY. Holomorphic curves with more than $n$ deficient curves in general position have a positive lower order.

Proof of Theorem 3. Let $\tilde{x}=\left(x_{0}, x_{1}, \ldots, x_{n}\right): \mathbf{C} \rightarrow \mathbf{C}^{n+1}-\{0\}$ and $\tilde{\alpha}^{(k)}=\left(\alpha_{0}^{(k)}, \alpha_{1}^{(k)}, \ldots, \alpha_{n}^{(k)}\right): \mathbf{C} \rightarrow\left(\mathbf{C}^{n+1}\right)^{*}-\{0\}$ be reduced representations of $x$ 
and $\alpha^{(k)}$, respectively. Then we can choose a nonzero complex number $\beta$ such that a reduced representation of a constant curve $\alpha^{(n+2)}: \mathbf{C} \rightarrow\left(P_{n} \mathbf{C}\right)^{*}$ is $\tilde{\alpha}^{(n+2)}=$ $\left(1, \beta, \beta^{2}, \ldots, \beta^{n}\right),\left\{\alpha^{(k)}\right\}_{k=1}^{n+2}$ is in general position, and $x$ is nondegenerate with respect to $\left\{\alpha^{(k)}\right\}_{k=1}^{n+2}$.

We now consider the holomorphic curve $y: \mathbf{C} \rightarrow P_{n} \mathbf{C}$ which Mori [9] constructed from $x$ and $\left\{\alpha^{(k)}\right\}_{k=1}^{n+2}$. We put

$$
F_{k}(z)=\sum_{j=0}^{n} \alpha_{j}^{(k)}(z) x_{j}(z)
$$

Let $G=\operatorname{det}\left\{\alpha_{j}^{(k)}\right\}_{0 \leq j \leq n, 1 \leq k \leq n+1}, G_{k}$ be the determinant of the matrix replaced the $k$ th column vector ${ }^{\mathrm{t}}\left(\alpha_{0}^{(k)}, \ldots, \alpha_{n}^{(k)}\right)$ of the matrix $\left\{\alpha_{j}^{(k)}\right\}_{0 \leq j \leq n, 1 \leq k \leq n+1}$ by the column vector ${ }^{\mathrm{t}}\left(\alpha_{0}^{(n+2)}, \ldots, \alpha_{n}^{(n+2)}\right)$, and $g_{k}=G_{k} / G$. We denote by $h(z)$ a common factor among $g_{1}(z) F_{1}(z), \ldots, g_{n}(z) F_{n}(z)$ and $g_{n+1}(z) F_{n+1}(z)$ up to nonvanishing entire functions such that $y_{k-1}(z)=g_{k}(z) F_{k}(z) / h(z)(k=1, \ldots, n+$ 1) are entire. Then, by the reasoning of Mori $\left[9\right.$, proof ${ }^{1}$ of Theorem 3.4], the holomorphic curve $y: \mathbf{C} \rightarrow P_{n} \mathbf{C}$ induced by $\tilde{y}=\left(y_{0}, y_{1}, \ldots, y_{n}\right): \mathbf{C} \rightarrow \mathbf{C}^{n+1}-\{0\}$ has the properties

$$
T(r, x) \sim T(r, y) \quad(r \rightarrow \infty)
$$

and, for $k=1,2, \ldots, n+1$,

$$
N\left(r, 0, y_{k-1}\right)=N\left(r, 0, F_{k}\right)+o(T(r, x)) \quad(r \rightarrow \infty) .
$$

Now we prove (5.2) and (5.3). Hadamard's inequality implies

$$
\left|G_{k}\right| \leq B \prod_{j=1, \neq k}^{n+1}\left|\tilde{\alpha}^{(j)}\right|
$$

where $B=\left|\tilde{\alpha}^{(n+2)}\right|$ is a constant. Using $\left|F_{k}\right| \leq|\tilde{x}|\left|\tilde{\alpha}^{(k)}\right|$, together with (5.4), we have

so

$$
\left|y_{k-1}\right|=\left|\frac{G_{k} F_{k}}{h G}\right| \leq B|\tilde{x}| \prod_{j=1}^{n+1} \frac{\left|\tilde{\alpha}^{(j)}\right|}{|h G|}
$$

$$
|\tilde{y}| \leq(n+1)^{1 / 2} B|\tilde{x}| \prod_{j=1}^{n+1} \frac{\left|\tilde{\alpha}^{(j)}\right|}{|h G|} .
$$

Hence, we have, with a suitable constant $K_{1}$,

$$
T(r, y) \leq T(r, x)+\sum_{j=1}^{n+1} T\left(r, \alpha^{(j)}\right)-N(r, 0, G)-N(r, 0, h)+N(r, \infty, h)+K_{1}
$$

Since $N(r, \infty, h) \leq N(r, 0, G)$, we obtain

$$
T(r, y) \leq T(r, x)+\sum_{j=1}^{n+1} T\left(r, \alpha^{(j)}\right)+K_{1}
$$

\footnotetext{
${ }^{1}$ There is a mistake in Mori's proof of his Theorem 3.4 [9], but he provides a correct proof in the "Correction to [9]". Unfortunately, his correction will not appear in the published paper, so we prove (5.2) and (5.3) along the lines of his correct proof.
} 
On the other hand, it follows from

$$
G_{k} \sum_{j=0}^{n} \alpha_{j}^{(k)} x_{j}=G h y_{k-1} \quad(k=1, \ldots, n+1)
$$

that $x_{j}=G h H_{j} / H$, where $H=\operatorname{det}\left\{G_{k} \alpha_{j}^{(k)}\right\}_{1 \leq k \leq n+1,0 \leq j \leq n}$ and $H_{j}$ is the determinant of the matrix obtained by replacing the $j$ th column vector

$$
{ }^{\mathrm{t}}\left(G_{1} \alpha_{j}^{(1)}, \ldots, G_{n+1} \alpha_{j}^{(n+1)}\right)
$$

of the matrix $\left\{G_{k} \alpha_{j}^{(k)}\right\}_{1 \leq k \leq n+1,0 \leq j \leq n}$ by the column vector ${ }^{\mathrm{t}}\left(y_{0}, \ldots, y_{n}\right)$. We put $\tilde{\eta}_{k}={ }^{\mathrm{t}}\left(G_{1} \alpha_{k}^{(1)}, \ldots, G_{n+1} \alpha_{k}^{(n+1)}\right)$. Then using Hadamard's inequality together with (5.4), we have

$$
\left|H_{j}\right| \leq|\tilde{y}| \prod_{k=0, \neq j}^{n}\left|\tilde{\eta}_{k}\right|
$$

and

$$
\left|\tilde{\eta}_{k}\right|^{2}=\sum_{l=1}^{n+1}\left|G_{l} \alpha_{k}^{(l)}\right|^{2} \leq \sum_{l=1}^{n+1}\left(B \prod_{j=1, \neq l}^{n+1}\left|\tilde{\alpha}^{(j)}\right|\right)^{2}\left|\tilde{\alpha}^{(l)}\right|^{2}=(n+1) B^{2} \prod_{j=1}^{n+1}\left|\tilde{\alpha}^{(j)}\right|^{2}
$$

so

$$
\left|H_{j}\right| \leq|\tilde{y}|\left\{(n+1)^{1 / 2} B \prod_{k=1}^{n+1}\left|\tilde{\alpha}^{(k)}\right|\right\}^{n}
$$

By Hadamard's inequality we also have

$$
|G| \leq \prod_{k=1}^{n+1}\left|\tilde{\alpha}^{(k)}\right|
$$

Hence,

$$
\begin{aligned}
\log |\tilde{x}| & =\log \left(\sum_{j=0}^{n}\left|\frac{h G H_{j}}{H}\right|^{2}\right)^{1 / 2} \\
& =\log |G|+\log \left(\sum_{j=0}^{n}\left|H_{j}\right|^{2}\right)^{1 / 2}+\log |h|-\log |H| \\
& \leq \log |\tilde{y}|+(n+1) \sum_{k=1}^{n+1} \log \left|\tilde{\alpha}^{(k)}\right|+\log |h|-\log |H|+\log B^{n}(n+1)^{(n+1) / 2},
\end{aligned}
$$

so, with a suitable constant $K_{2}$,

$$
\begin{aligned}
T(r, x) \leq & T(r, y)+(n+1) \sum_{k=1}^{n+1} T\left(r, \alpha^{(k)}\right)+N(r, 0, h) \\
& -N(r, \infty, h)-N(r, 0, H)+K_{2} .
\end{aligned}
$$

Since $N(r, 0, h) \leq N(r, 0, H)$, we obtain

$$
T(r, x) \leq T(r, y)+(n+1) \sum_{k=1}^{n+1} T\left(r, \alpha^{(k)}\right)+K_{2} .
$$


Next (5.4) and (5.6) imply

$$
N\left(r, 0, G_{k}\right) \leq \sum_{j=0, \neq k}^{n} T\left(r, \alpha^{(j)}\right)+K_{3}
$$

and

$$
N(r, 0, G) \leq \sum_{j=0}^{n} T\left(r, \alpha^{(j)}\right)+K_{4},
$$

where $K_{3}$ and $K_{4}$ are constants. Hence, using (5.8) we have

$$
\begin{aligned}
N\left(r, 0, y_{k-1}\right) & \leq N\left(r, 0, F_{k}\right)+N\left(r, 0, G_{k}\right) \\
& \leq N\left(r, 0, F_{k}\right)+\sum_{j=0, \neq k}^{n} T\left(r, \alpha^{(j)}\right)+K_{4} .
\end{aligned}
$$

Since $|H| \leq\left(\prod_{j=1}^{n+1}\left|G_{j}\right|\right)|G|$, we have

$$
N(r, 0, H) \leq N(r, 0, G)+\sum_{j=1}^{n+1} N\left(r, 0, G_{k}\right)+K_{5}
$$

where $K_{5}$ is a constant. Hence, using $N(r, 0, h) \leq N(r, 0, H)$, together with (5.8), (5.9), and (5.11), we have

$$
\begin{aligned}
N\left(r, 0, F_{k}\right) & \leq N\left(r, 0, y_{k-1}\right)+N(r, 0, G)+N(r, 0, h) \\
& \leq N\left(r, 0, y_{k-1}\right)+2 N(r, 0, G)+\sum_{j=1}^{n+1} N\left(r, 0, G_{j}\right)+K_{5} \\
& \leq N\left(r, 0, y_{k-1}\right)+(n+2) \sum_{j=1}^{n+1} T\left(r, \alpha^{(j)}\right)+K_{6},
\end{aligned}
$$

where $K_{6}$ is a suitable constant. Therefore using (5.1) in (5.5), (5.7), (5.10), and (5.12), we obtain (5.2) and (5.3).

Now it follows from (5.2) and (5.3) that

$$
\delta\left(\alpha^{(k)}, x\right)=1-\limsup _{r \rightarrow \infty} \frac{N\left(r, 0, y_{k-1}\right)}{T(r, y)}=\delta\left(a^{(k)}, y\right) \quad(k=1,2, \ldots, n+1),
$$

where $a^{(k)} \in\left(P_{n} C\right)^{*}$ are induced by $(0, \ldots, 0,1,0, \ldots, 0)((k-1)$ th spot $)$. Therefore, Theorem 3 follows Theorem 2 in Toda [14].

6. Proof of Theorem 1(II). We may assume that $\delta\left(\alpha^{(k)}\right) \geq 1-\cos \pi \mu, k=$ $1,2, \ldots, n$. Then

$$
(4 / \mu) \sin ^{-1}\left(\delta\left(\alpha^{(k)}\right) / 2\right)^{1 / 2} \geq 2 \pi
$$

so

$$
\min \left\{2 \pi,(4 / \mu) \sin ^{-1}\left(\delta\left(\alpha^{(k)}\right) / 2\right)^{1 / 2}\right\}=2 \pi, \quad k=1, \ldots, n .
$$

Hence, it follows from Lemma 5 that

$$
\sum_{k=n+1}^{q} \min \left\{2 \pi, \frac{4}{\mu} \sin ^{-1}\left(\frac{\delta\left(\alpha^{(k)}\right)}{2}\right)^{1 / 2}\right\}=0
$$


and, consequently,

$$
\delta\left(\alpha^{(k)}\right)=0, \quad k=n+1, \ldots, q .
$$

We next assume that $\delta\left(\alpha^{(k)}\right)>1-\cos \pi \mu(k=1, \ldots, p ; 0 \leq p<n)$ and $\delta\left(\alpha^{(k)}\right) \leq 1-\cos \pi \mu(k=p+1, \ldots, q)$. Then it follows from Lemma 5 that

$$
\sum_{k=p+1}^{q} \frac{4}{\mu} \sin ^{-1}\left(\frac{\delta\left(\alpha^{(k)}\right)}{2}\right)^{1 / 2} \leq 2(n-p) \pi .
$$

Hence, from the reasoning of $\S 3$ of Edrei [5] we can deduce that

$$
\sum_{k=p+1}^{q} \delta\left(\alpha^{(k)}\right) \leq(n-p)(1-\cos \pi \mu) .
$$

It follows from Lemma 1 of Edrei [5] that equality in (6.1) is possible if and only if exactly $n-p$ of $\left\{\delta\left(\alpha^{(k)}\right)\right\}_{k=p+1}^{q}$ are equal to $1-\cos \pi \mu$ and all other $\delta\left(\alpha^{(k)}\right)$ are equal to zero.

Thus the proof of Theorem 1(II) is complete.

7. Proof of Theorem 1(III). Our assumption $\mu>1 / 2$ implies

$$
(4 / \mu) \sin ^{-1}\left(\delta\left(\alpha^{(k)}\right) / 2\right)^{1 / 2}<2 \pi,
$$

so it follows from Lemma 5 that

$$
\sum_{k=1}^{q} \frac{4}{\mu} \sin ^{-1}\left(\frac{\delta\left(\alpha^{(k)}\right)}{2}\right)^{1 / 2} \leq 2 n \pi .
$$

Hence, from the reasoning of $\S 2$ of Edrei [5] we can deduce that

$$
\sum_{k=1}^{q} \delta\left(\alpha^{(k)}\right) \leq[2 n \mu]+1-\cos (2 n \mu-[2 n \mu])(\pi / 2),
$$

which proves Theorem 1 (III).

\section{REFERENCES}

1. A. Baernstein II, Proof of Edrei's spread conjecture, Proc. London Math. Soc. (3) 26 (1973), 418-434.

2. __ Integral means, univalent functions and circular symmetrization, Acta Math. 133 (1974), 139-169.

3. C.-T. Chuang, Une généralisation d'une inégalité de Nevanlinna, Sci. Sinica 13 (1964), 887-895.

4. J. Dufresnoy, Sur les valeurs exceptionnelles des fonctions méromorphes voisines d'une fonction méromorphe donnée, C. R. Acad. Sci. Paris Sér. A-B 208 (1939), 255-257.

5. A. Edrei, Solution of the deficiency problem for functions of small lower order, Proc. London Math. Soc. (3) 26 (1973), 435-445.

6. A. Edrei and W. H. J. Fuchs, On the growth of meromorphic functions with several deficient values, Trans. Amer. Math. Soc. 93 (1959), 292-328.

7. A. V. Krytov, Deficiencies of entire functions of finite lower order, Ukrain. Math. J. 31 (1979), 210-214.

8. S. Mori, Sum of deficiencies and the order of a meromorphic function, Tôhoku Math. J. (2) 2 (1970), 659669.

9. __ Remarks on holomorphic mappings, Contemporary Math. 25 (1983), 101-113.

10. R. Nevanlinna, Le théorème de Picard-Borel et la théorie des fonctions méromorphes, GauthierVillars, Paris, 1929; reprint, Chelsea, New York, 1974. 
11. K. Niino, Spread relation and value distribution in an angular domain of holomorphic curves, Kōdai Math. Sem. Rep. 28 (1977), 361-371.

12. B. Shiffman, New defect relations for meromorphic functions on $C^{n}$, Bull. Amer. Math. Soc. (N.S.) 7 (1983), 599-601.

13. W. Stoll, Value distribution theory for moving targets, Lecture at Res. Inst. for Math. Sci., Kyoto Univ., 1983.

14. N. Toda, Sur la croissance de fonctions algébroïdes a valeurs déficientes, Kōdai Math. Sem. Rep. 22 (1970), 324-337.

15. H. Weyl and J. Weyl, Meromorphic functions and analytic curves, Ann. of Math. Stud., no. 12, Princeton Univ. Press, Princeton, N.J., 1943; reprint, Kraus, New York, 1965.

16. H.-H. Wu, The equidistribution theory of holomorphic curves, Ann. of Math. Stud., no. 64, Princeton Univ. Press, Princeton, N.J.; Univ. of Tokyo Press, Tokyo, 1970.

17. L. Yang, Deficient functions of meromorphic functions, Sci. Sinica 24 (1981), 1179-1189.

Faculty of Technology, Kanazawa University, 2-40-20, Kodatsuno, KanaZAWA 920, JAPAN 